\title{
Real-time biodetection using a smartphone-based dual-color surface plasmon resonance sensor
}

Qiang Liu

Huizhen Yuan

Yun Liu

Jiabin Wang

Zhenguo Jing

Wei Peng 


\title{
Real-time biodetection using a smartphone-based dual-color surface plasmon resonance sensor
}

\author{
Qiang Liu, Huizhen Yuan, Yun Liu, Jiabin Wang, Zhenguo Jing, ${ }^{*}$ and Wei Peng* \\ Dalian University of Technology, College of Physics and Optoelectronic Engineering, Dalian, China
}

\begin{abstract}
We proposed a compact and cost-effective red-green dual-color fiber optic surface plasmon resonance (SPR) sensor based on the smartphone. Inherent color selectivity of phone cameras was utilized for realtime monitoring of red and green color channels simultaneously, which can reduce the chance of false detection and improve the sensitivity. Because there are no external prisms, complex optical lenses, or diffraction grating, simple optical configuration is realized. It has a linear response in a refractive index range of 1.326 to 1.351 $\left(R^{2}=0.991\right)$ with a resolution of $2.3 \times 10^{-4}$ RIU. We apply it for immunoglobulin $\mathrm{G}$ (IgG) concentration measurement. Experimental results demonstrate that a linear SPR response was achieved for IgG concentrations varying from 0.02 to $0.30 \mathrm{mg} / \mathrm{ml}$ with good repeatability. It may find promising applications in the fields of public health and environment monitoring owing to its simple optics design and applicability in real-time, label-free biodetection. @ 2018 Society of Photo-Optical Instrumentation Engineers (SPIE) [DOI: 10.1117/1.JBO.23.4.047003]
\end{abstract}

Keywords: optical fiber sensor; surface plasmon resonance; biological sensing and sensors; visibility and imaging.

Paper 170798R received Dec. 10, 2017; accepted for publication Mar. 30, 2018; published online Apr. 27, 2018.

\section{Introduction}

Cost-effective, portable, and rapid sensing systems play critical roles in bringing biodetection technologies from central laboratories to field applications. ${ }^{1-3}$ Although laboratory-based benchtop instruments usually provide higher sensitivity and stability, they are usually expensive and bulky. Nowadays, there are intense interests in converting the smartphone into a low-cost and user-friendly sensing tool for different biochemical sensing applications. It is expected to be applied in situations where laboratory facilities are not available, such as point-of-care analysis in the home, clinic, or remote locations. Real-time communication and data sharing from any remote location are possible with the help of smartphones. Examples include smartphone-based microscopy, ${ }^{4}$ enzyme-linked immunosorbent assay (ELISA), ${ }^{5}$ polymerase chain reaction (PCR) ${ }^{6}$ and surface plasmon resonance (SPR). ${ }^{7,8}$ Unlike ELISA or PCR, SPR does not require a labeling procedure, which is a great advantage in field applications. $^{9-11}$ Utilizing high-performance complementary metal-oxide-semiconductor (CMOS)-based cameras on smartphones, many groups have designed spectrum- and intensitybased SPR biosensors on smartphone platforms. For spectrum-based assays, smartphones are usually converted to simple spectrometers. The changes of spectrum mean some reaction has happened or some analytes are present. Zhang et al. ${ }^{12}$ demonstrated a grating-coupled SPR smartphone biosensor, which relies on a disposable sensor chip with Au diffraction grating and a compact disk as the spectra dispersive unit. Although it does not contain electronic components, the use of apertures, polarizers, and diffraction gratings increases the complexity of the optical configuration. A portable spectrum-based fiber optic glycerol smartphone SPR sensor was demonstrated by Bremer and Roth. ${ }^{13}$ Silver-coating fiber SPR sensor eliminates the need

*Address all correspondence to: Zhenguo Jing, E-mail: jingzg@ dlut.edu.cn; Wei Peng, E-mail: wpeng@dlut.edu.cn for external prisms, optical lenses, etc. for easier optical coupling and optical alignment. Spectrum-based analysis usually means more information and higher sensitivity, but it also means more complex image processing algorithms and greater computational complexity. Smartphones are only used to capture images, and subsequent analysis needs to be performed on the computer, which limited their actual diagnostic usefulness. Intensity-based assay has attracted much attention because of its low-cost, simplicity, and practicality. It provides real-time data analysis and is usually stable, inexpensive, and simple to perform. The optical intensity at fixed angle or wavelength is monitored by smartphones in real time. Preechaburana et al. ${ }^{14}$ and Guner et al. ${ }^{15}$ constructed angle- and wavelengthresolved intensity-based smartphone SPR sensors, respectively. Combined with fiber optic sensing technology, Liu et al. ${ }^{7}$ proposed a fiber optic smartphone SPR sensor based on intensity integration method, which greatly simplified the optical configuration. In addition to the advantages of label-free, realtime analysis, it can be easily multiplexed to enable highthroughput screening in an array format.

In this paper, we propose a smartphone-based dual-color fiber optic SPR sensor. Red and green color channels are monitored simultaneously based on the inherent color selectivity of phone cameras instead of using discrete lenses or diffraction gratings to get spectral data as those spectrum-based SPR sensors reported to date. Also, it does not require additionally external monochromatic light source or narrowband filter as those intensity-based single-color SPR sensors, which greatly simplify the system configuration and lower the cost. $\mathrm{Ag} / \mathrm{Au}$ bilayer film was implemented to enhance sensitivity and color contrast. A nakedeye observable color change can be seen from the images corresponding to the different refractive index (RI). A schematic of the dual-color fiber optic SPR sensor developed here is shown in

$1083-3668 / 2018 / \$ 25.00$ @ 2018 SPIE 


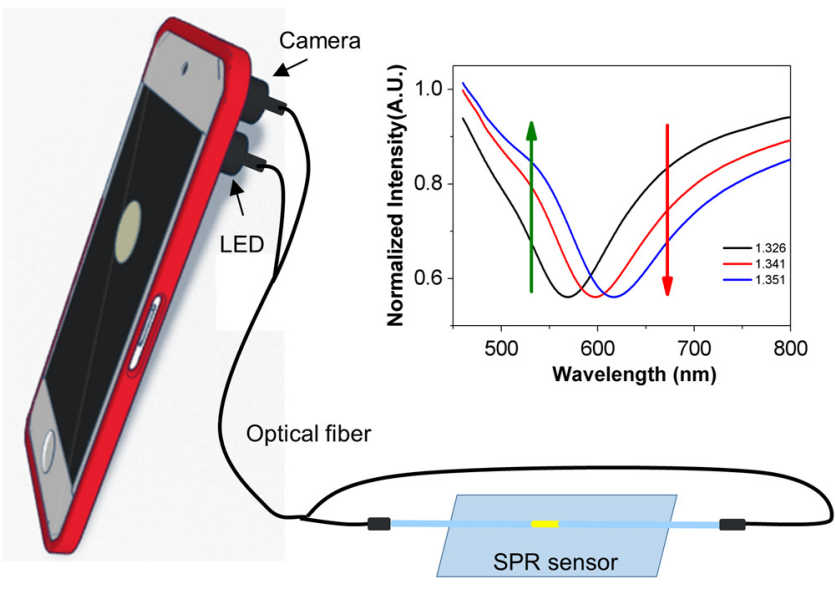

Fig. 1 Schematic diagram of smartphone-based dual-color fiber optic SPR sensor. (Inset) Normalized spectrums of the sensor in different RIs obtained by spectrometer.

Fig. 1. This method quantifies SPR signals from two color channels (one decreases, while the other increases as the surrounding RI changes) to enhance the accuracy and sensitivity of detection. An Android application software was developed to analyze the red-green-blue (RGB) image data in real time. SPR sensor was functionalized with staphylococcal protein A (protein A) for specific identification and binding of immunoglobulin $\mathrm{G}(\mathrm{IgG})$. Experimental results indicate that the proposed dual-color SPR sensor is effective for biosensor applications.

\section{Red-Green Dual-Color Fiber Optic SPR Sensor}

$\mathrm{Au}$ and $\mathrm{Ag}$ are the most widely used metal for the SPR experiments. ${ }^{16}$ Ag metal-based SPR sensors usually have higher sensitivity and smaller line width of resonant dip compared with Au mental-based SPR sensors; ${ }^{17-19}$ however, they are highly prone to oxidation, which greatly restricts their application. Coating $\mathrm{Au}$ film above the $\mathrm{Ag}$ film to eliminate the oxidation has been extensively studied. ${ }^{20-22}$ In this work, we employed $\mathrm{Ag} / \mathrm{Au}$ bilayer film to enhance sensitivity and color contrast. We used hard polymer cladding optical fiber (HPCF, HP 400/430-37/730E, YOFC Inc., Wuhan, China) to fabricate the
SPR sensor. Diameters of the fiber core and cladding were 400 and $430 \mu \mathrm{m}$, with numerical aperture 0.37 . The fiber optic SPR sensor was 6-cm long, and in the middle of the fiber existed a 5-mm sensing region with cladding removed. The sensing region was cleaned by acetone and deionized water subsequently, and then coated with $\mathrm{Ag} / \mathrm{Au}$ bilayer film (25-nm Ag layer and 15-nm Au layer). A magnetron sputtering coater (K575XD from E. M. Technologies Ltd., Kent) was used for metal layer coating. It features a rotating sample table that ensures even depositions. The rotation speed is about $6 \mathrm{r} / \mathrm{min}$. To get a metal film deposited around on the fiber core surface uniformly, we used a set of rotation clamping mechanisms to make the fiber optic sensing part rotate uniformly in the vacuum chamber. Thickness monitoring was achieved by quartz crystal oscillation method.

A homemade wavelength interrogation fiber optic SPR system shown in Fig. 2(a) was utilized to demonstrate the performance of the fabricated SPR sensor. It consists of a halogen lamp (HL-2000-FHSA, Ocean Optics, Inc.) and a high-resolution spectrometer (HR4000, Ocean Optics, Inc.). The fabricated sensor was connected to the devices through SMA905 connectors. Figure 2(b) shows a clear SPR dip that shifts toward the red wavelength range for increasing RI of the surrounding liquid. We changed the liquid RIs using different sodium chloride solutions and calibrated the RI values by an Abbe refractometer (WAY-2S). Through linear fitting, the sensitivity of measuring $\mathrm{RI}$ of the sensor could reach $1839 \mathrm{~nm} / \mathrm{RIU}\left(R^{2}=0.992\right)$.

The intensity at a fixed wavelength will change along with the SPR curve shifting caused by variations of RI. When monitoring the intensity at two wavelengths across the dips, it can be observed that the intensity for shorter wavelength increases and that for longer wavelength decreases. By taking the difference between the normalized intensity at two wavelengths, almost twofold improvement in sensitivity can be expected. A miniaturized optical waveguide SPR sensor based on dual light-emitting diodes as light sources has been demonstrated by Suzuki et al. $^{23}$ In this work, we used the inherent color selectivity of phone cameras to monitor red and green color channels simultaneously. CMOS digital cameras on the smartphone (HTC D816d) served as detector and the flashlight served as light source. Light intensities for red and green color channels were obtained simultaneously within the RGB passbands of the internal color filters of digital camera. ${ }^{24}$
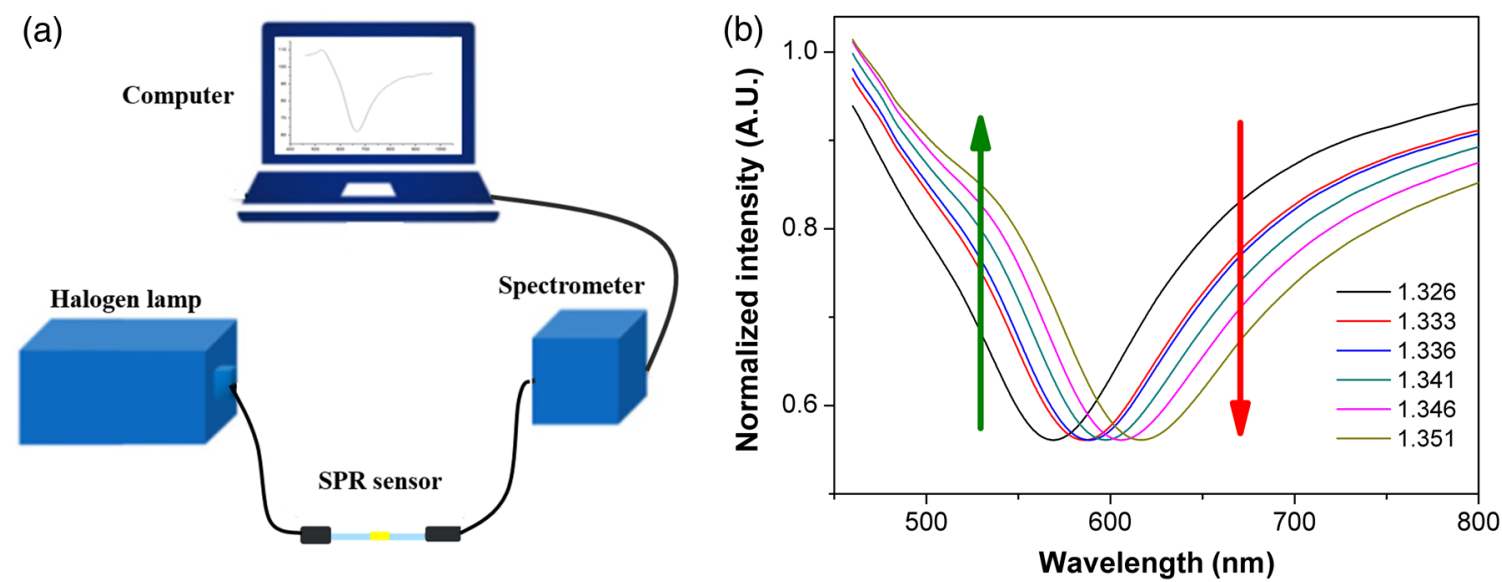

Fig. 2 Spectral characteristics of fiber optic SPR sensor: (a) wavelength interrogation system used to verify the fabricated SPR sensors and (b) normalized spectrums of the sensor in different Rls ranging from 1.326 to 1.351 . 
We developed an Android application for real-time image processing. There are 24 bits per pixel in color images, which include three 8-bit integers ( 0 to 255 ) that indicate the intensity of RGB colors. R values and $\mathrm{G}$ values of all the pixels in the region of interest (ROI) are averaged as the intensity of red channel and green channel, respectively. After capturing the required images with $640 \times 480$ resolution, the average intensities of red and green channels were calculated and displayed on the screen in real time. In this work, a rectangular area covering the bright image of optical fiber's end face was chosen as the ROI. Each recorded intensity was typically averaged over 2 s (10 averages) to reduce the noise.

Responses of red and green channels upon sequential injection of sodium chloride solutions at increasing RI (1.326 to 1.351) are presented in Fig. 3. Figure 3(a) shows the realtime color images when the sensor region was immersed in different RI solutions. A naked-eye observable color change can be seen from the images. The intensities of each channel are normalized with respect to the intensities when the sensor is exposed to the air, and the normalized intensity variations of green channel and red channel are shown in Fig. 3(b). To obtain reliable data, the test for each solution was performed three times. Error bars indicate the standard deviation (SD) of the measurements. As RI increases, the green channel intensity increases and red channel intensity decreases. In this work, we define the differential normalized intensity between green and red channels as dual-color sensor response.

Normalized intensity shifts of dual-color channel are shown in Fig. 4. Error bars correspond to the SD of triplicate measurements. It illustrates that the normalized intensity shift as a function of RI can be linear fitted in the RI range of 1.326 to 1.351 , which can be used for many biochemical samples measurement. ${ }^{25,26}$ In the RI range of 1.326 to 1.351 , the system provides a sensitivity of $11.542 /$ RIU. Analysis of the baseline noise was also performed. It was determined that the SD of noise in the normalized intensity shift is $2.6 \times 10^{-3}$; so, the RI resolution can be calculated to be $2.3 \times 10^{-4}$ RIU.
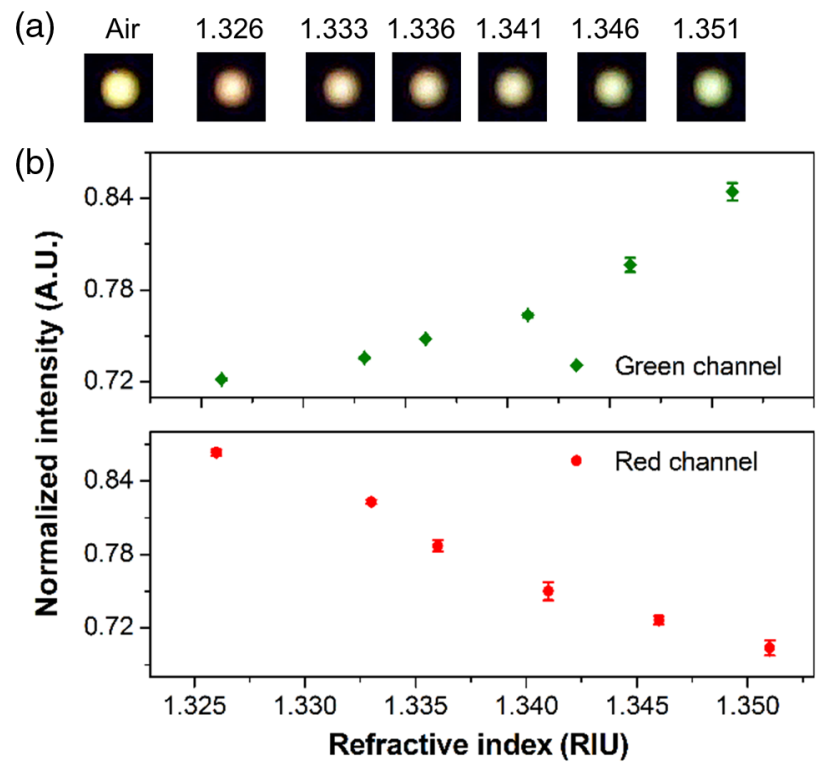

Fig. 3 Responses of red and green channels upon sequential injection of sodium chloride solutions at increasing RI (1.326 to 1.351): (a) real-time images and (b) normalized intensities of each channel.

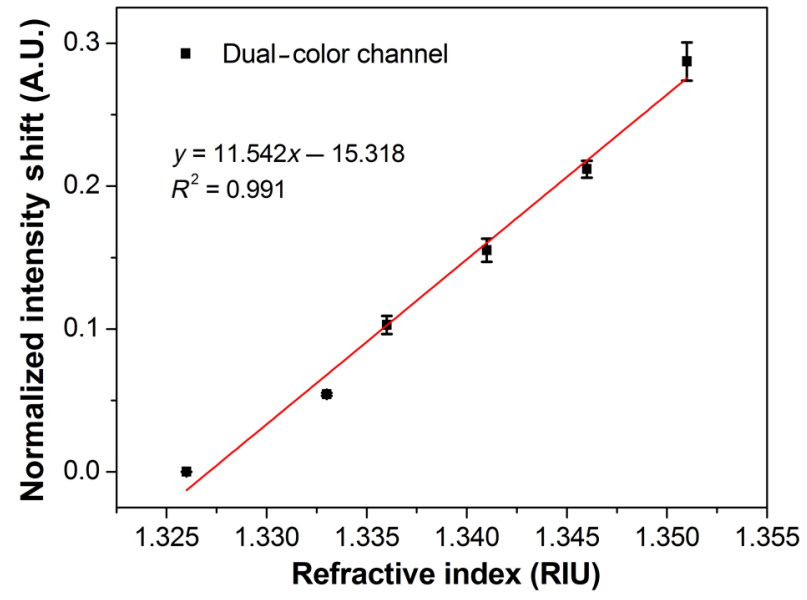

Fig. 4 Plots of normalized intensity shifts as a function of RI ranging from 1.326 to 1.351 .

\section{Real-Time IgG Detection}

To confirm the feasibility of this dual-color SPR sensor in biosensing applications, the protein $\mathrm{A}$ and rabbit IgG interaction was monitored. Protein A interacts with the Fc region of human, rabbit, pig, and guinea pig IgG with high affinity and specificity. ${ }^{27}$ In this work, we used protein A-modified SPR sensor for concentration detection of rabbit IgG.

Sensor functionalization and protein A-IgG binding procedure are shown in Fig. 5. An amine-coupling reaction was used to facilitate the attachment of protein A. The sensor was soaked in an ethanolic solution of 11-mercaptoundecanoic acid $(1 \mathrm{mmol} / \mathrm{L})$ at $25^{\circ} \mathrm{C}$ for $2 \mathrm{~h}$ to form a carboxyl surface, and then immersed into an aqueous solution containing 1ethyl-3-(3-dimethylamino-propyl) carbodiimide hydrochloride $(0.55 \mathrm{~mol} / \mathrm{L})$ and $\mathrm{N}$-hydroxysuccinimide $(0.5 \mathrm{~mol} / \mathrm{L})$ for $30 \mathrm{~min}$ at $4^{\circ} \mathrm{C}$ to convert the carboxyl surface to activated ester. The treated sensor was dipped in protein $\mathrm{A}[0.1 \mathrm{mg} / \mathrm{mL}$ in 0.01-mol/L phosphate-buffered solution (PBS; $\mathrm{pH}=7.4$ )] for $30 \mathrm{~min}$. Unreacted ester groups were blocked by bovine serum albumin $(1 \mathrm{mg} / \mathrm{mL}$ in PBS) for $15 \mathrm{~min}$.

A mini pump and a homemade flow cell were used for sample injection. The flow rate is about $250 \mu \mathrm{L} / \mathrm{min}$. Real-time responses of $\mathrm{IgG}$ detection are shown in Fig. 6. Figure 6(a) shows the responses of green and red channels, and Fig. 6(b) shows the calibrated responses of dual-color channel. PBS buffer was pumped into the flow cell at the very beginning to obtain a stable baseline. When the baseline was stable, we started the real-time biodetection of different $\operatorname{IgG}$ samples. Multiple measurements on one sensor were possible using regeneration solutions. Acetic acid solution ( $\mathrm{pH} 2.0)$ was pumped into the flow cell at a flow rate of $250 \mu \mathrm{L} /$ min for 5 min for surface regeneration. After regeneration, the baseline returned to its initial value. There was no clear deterioration (SD of baseline difference $=1.6 \times 10^{-3}$ ) during 10 binding and regeneration cycles. Figure 6(c) shows real-time binding process of five different $\mathrm{IgG}$ concentrations, and $\mathrm{IgG}$ samples with various concentrations ranging from 0.02 to $0.30 \mathrm{mg} / \mathrm{mL}(0.02$, $0.05,0.10,0.15$, and $0.30 \mathrm{mg} / \mathrm{mL}$ ) were pumped into the flow cell for $8 \mathrm{~min}$. After the specific recognition and binding stage, PBS buffer was used to wash away the unbound IgG from the surface. Signals from the 20th minute to the 22nd minute are averaged as the sensor response corresponding to the specific concentration. To get reliable data, the tests were performed 


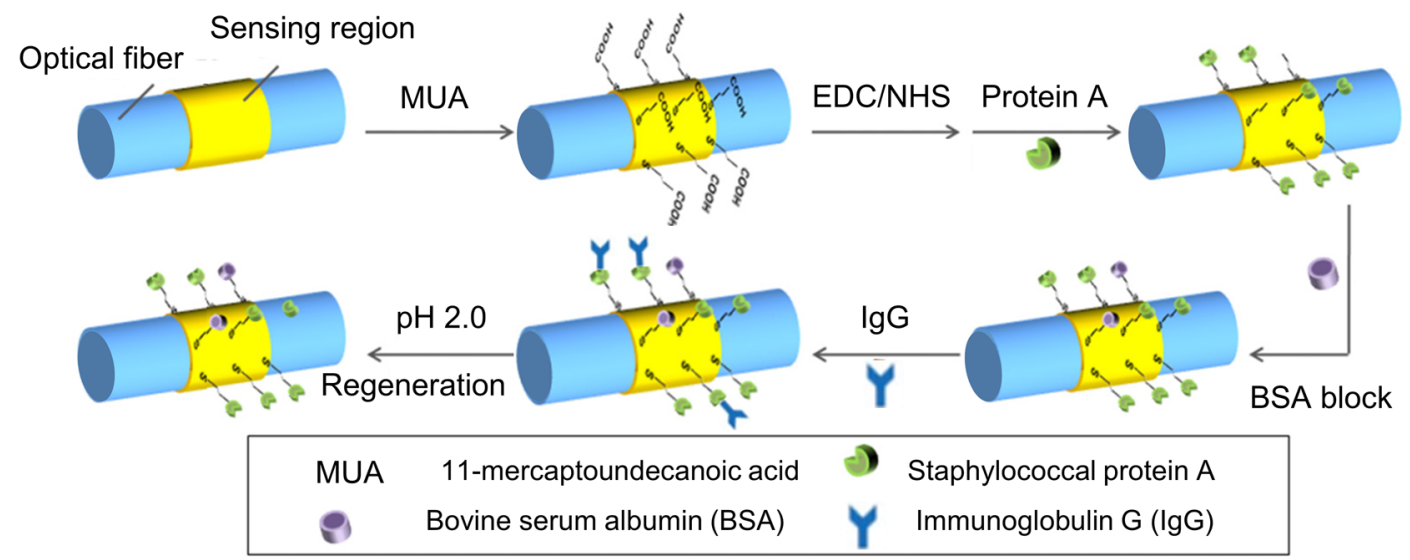

Fig. 5 Schematic illustration of functionalization and protein A-lgG binding procedure of the SPR sensor.

(a)

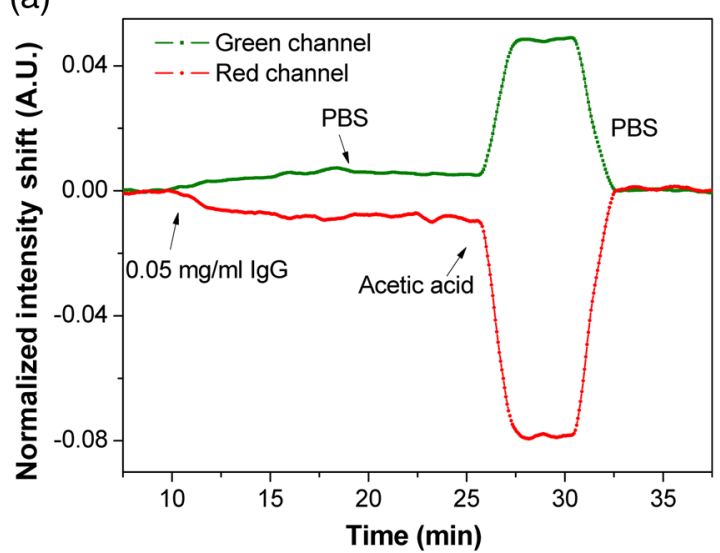

(c)

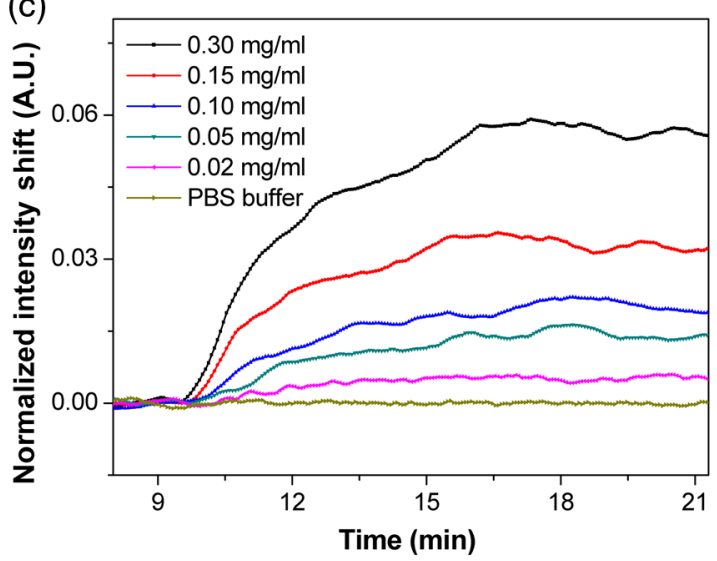

(b)

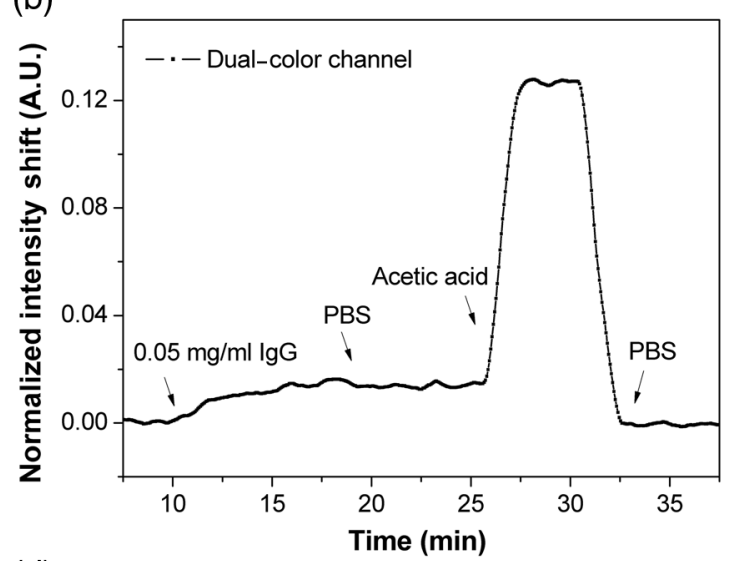

(d)

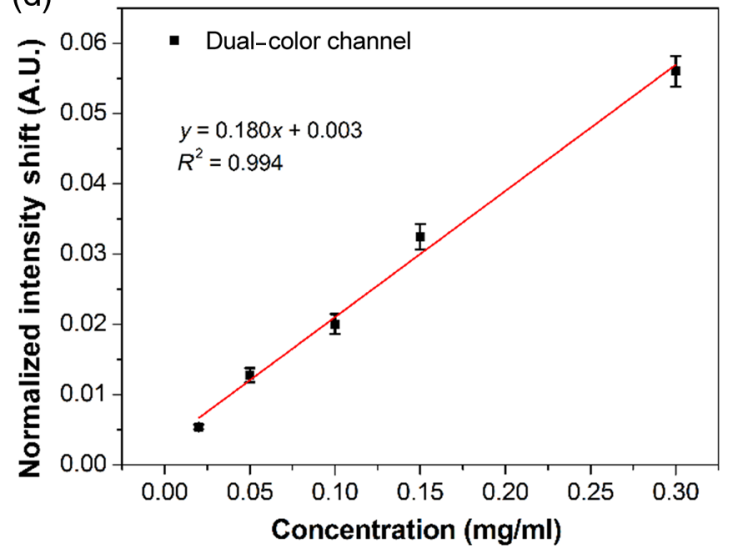

Fig. 6 Real-time biodetection with various concentrations of IgG samples: (a) response curves of green and red channels for $0.05-\mathrm{mg} / \mathrm{ml} \mathrm{IgG} \mathrm{sample,} \mathrm{(b)} \mathrm{calibrated} \mathrm{response} \mathrm{curves} \mathrm{of} \mathrm{dual-color} \mathrm{channel} \mathrm{for}$ $0.05-\mathrm{mg} / \mathrm{ml} \mathrm{IgG} \mathrm{sample,} \mathrm{(c)} \mathrm{real-time} \mathrm{binding} \mathrm{process} \mathrm{of} \mathrm{five} \mathrm{different} \mathrm{concentrations} \mathrm{from} 0.02$ to $0.30 \mathrm{mg} / \mathrm{ml}$, and (d) plot of normalized intensity shift versus the IgG concentrations.

three times for each $\operatorname{IgG}$ concentration. There is a linear relationship between $\operatorname{IgG}$ concentrations and the normalized intensity shift $\left(R^{2}=0.994\right)$ as shown in Fig. 6(d). Error bars represent the SD of triplicate measurement. The relative SD was $<7 \%$ for each concentration, which shows its good stability.

The results indicate that this dual-color SPR sensor is effective for biosensor applications. We can further enhance its sensitivity and resolution by optimizing the performance of SPR sensor probe and utilizing secondary antibodies or nanoparticles to achieve signal amplification. ${ }^{28}$

\section{Conclusion}

A portable and low-cost dual-color SPR sensor has been successfully realized through simple optical configuration. In conjunction with inherent color selectivity of the smartphone camera, SPR signals from red and green channels were quantified in real time, which can reduce the chance of false detection 
and improve the sensitivity. It shows a linear response to RI changes between 1.326 and 1.351 with a resolution of $2.3 \times 10^{-4}$ RIU. We used the sensor for real-time biodetection by monitoring the binding of IgG and protein A. Experimental results demonstrate that a linear SPR response was achieved for IgG concentrations varying from 0.02 to $0.30 \mathrm{mg} / \mathrm{ml}$ with good repeatability. Considering the advantage of portability and simplicity, it is attractive for point-of-care and remote detection of biomedical and environmental targets.

\section{Appendix}

The code for the smartphone application can be found online at https://github.com/lqls/Dual_Color_SPR.

\section{Disclosures}

No conflicts of interest, financial or otherwise, are declared by the authors.

\section{Acknowledgments}

The authors would like to express thanks for financial support from the National Natural Science Foundation of China (Grant Nos. 61727816, 61520106013, 61705031, and 11474043) and the National Science Foundation for Postdoctoral Scientists of China (Grant No. 2017M610175).

\section{References}

1. D. Zhang et al., "Biosensors and bioelectronics on smartphone for portable biochemical detection," Biosens. Bioelectron. 75, 273-284 (2016).

2. J. Hu et al., "Portable microfluidic and smartphone-based devices for monitoring of cardiovascular diseases at the point of care," Biotechnol. Adv. 34(3), 305-320 (2016).

3. S. Kanchi et al., "Smartphone based bioanalytical and diagnosis applications: a review," Biosens. Bioelectron. 102, 136-149 (2018).

4. Y. L. Sung et al., "Fabricating optical lenses by inkjet printing and heatassisted in situ curing of polydimethylsiloxane for smartphone microscopy," J. Biomed. Opt. 20(4), 047005 (2015).

5. T. Ogirala et al., "Smartphone-based colorimetric ELISA implementation for determination of women's reproductive steroid hormone profiles," Med. Biol. Eng. Comput. 55(10), 1735-1741 (2017).

6. X. Qiu et al., "A smartphone-based point-of-care diagnosis of H1N1 with microfluidic convection PCR," Microsyst. Technol. 23(7), 2951-2956 (2017).

7. Y. Liu et al., "Surface plasmon resonance biosensor based on smart phone platforms," Sci. Rep. 5, 12864 (2015).

8. D. Gallegos et al., "Label-free biodetection using a smartphone," Lab Chip 13(11), 2124 (2013).

9. Y. Chen and H. Ming, "Review of surface plasmon resonance and localized surface plasmon resonance sensor," Photonic Sens. 2(1), 37-49 (2012).

10. V. Kodoyianni, "Label-free analysis of biomolecular interactions using SPR imaging," Biotechniques 50(1), 32-40 (2011).

11. H. H. Nguyen et al., "Surface plasmon resonance: a versatile technique for biosensor applications," Sensors (Basel, Switzerland) 15(5), 1048110510 (2015).

12. J. Zhang et al., "Lipopolysaccharides detection on a grating-coupled surface plasmon resonance smartphone biosensor," Biosens. Bioelectron. 99, 312-317 (2018).

13. K. Bremer and B. Roth, "Fibre optic surface plasmon resonance sensor system designed for smartphones," Opt. Express 23(13), 17179-17184 (2015).

14. P. Preechaburana et al., "Surface plasmon resonance chemical sensing on cell phones," Angew. Chem. Int. Ed. Engl. 51(46), 11585-11588 (2012).
15. H. Guner et al., "A smartphone based surface plasmon resonance imaging (SPRi) platform for on-site biodetection," Sens. Actuators, B 239, 571-577 (2017).

16. J. Homola, S. S. Yee, and G. Gauglitz, "Surface plasmon resonance sensors: review," Sens. Actuators, B 54(1), 3-15 (1999).

17. M. Mitsushio, Y. Abe, and M. Higo, "Sensor properties and surface characterization of silver-deposited SPR optical fibers," Anal. Sci. 26(9), 949-955 (2010).

18. M. Mitsushio, K. Miyashita, and M. Higo, "Sensor properties and surface characterization of the metal-deposited SPR optical fiber sensors with Au, Ag, Cu, and Al," Sens. Actuators, A 125(2), 296-303 (2006).

19. S. H. Kim et al., "The effect of Au/Ag bimetallic thin-films on surface plasmon resonance properties comparing with those of Au and Ag single thin-films," J. Nanosci. Nanotechnol. 18(3), 1777-1781 (2018).

20. S. Zynio et al., "Bimetallic layers increase sensitivity of affinity sensors based on surface plasmon resonance," Sensors 2(2), 62-70 (2002).

21. M. Lu et al., "Optimization of surface plasmon resonance biosensor with $\mathrm{Ag} / \mathrm{Au}$ multilayer structure and fiber-optic miniaturization," Plasmonics 12(3), 663-673 (2017).

22. C.-T. Li et al., "Ag/Au bi-metallic film based color surface plasmon resonance biosensor with enhanced sensitivity, color contrast and great linearity," Biosens. Bioelectron. 36(1), 192-198 (2012).

23. A. Suzuki et al., "Development of novel optical waveguide surface plasmon resonance (SPR) sensor with dual light emitting diodes," Sens. Actuators, B 106(1), 383-387 (2005).

24. E. H. Doeven et al., "Red-green-blue electrogenerated chemiluminescence utilizing a digital camera as detector," Anal. Chem. 86(5), 2727-2732 (2014).

25. H. H. Jeong et al., "Real-time label-free immunoassay of interferongamma and prostate-specific antigen using a fiber-optic localized surface plasmon resonance sensor," Biosens. Bioelectron. 39(1), 346-351 (2013).

26. J. F. Masson et al., "Dual Kretschmann and Otto configuration fiber surface plasmon resonance biosensor," Opt. Express 25(22), 26950 (2017).

27. N. L. Brown et al., "A study of the interactions between an IgG-binding domain based on the B domain of staphylococcal protein A and rabbit IgG," Rev. Mol. Biotechnol. 10(1), 9-16 (1998).

28. R. Liu et al., "Surface plasmon resonance biosensor for sensitive detection of microRNA and cancer cell using multiple signal amplification strategy," Biosens. Bioelectron. 87(Suppl. C), 433-438 (2017).

Qiang Liu received his ME degree in optical engineering from the School of Physics, Dalian University of Technology, Dalian, China, in 2017. His research area is focused on optical fiber sensors, time division multiplexing, and wavelength division multiplexing surface plasmon resonance sensor system.

Huizhen Yuan received her BE degree in material chemistry from Hebei University, Baoding, China, in 2014. Currently, she is pursuing her PhD in optical engineering at Dalian University of Technology. Her research area is focused on fiber-optic glucose sensors and surface plasmon resonance sensors.

Yun Liu received his $\mathrm{PhD}$ in optical engineering from the School of Physics, Dalian University of Technology, Dalian, China, in 2016. Currently, he is working in the School of Physics at Dalian University of Technology as a lecturer. His research interests are new photonic devices, fiber-optic sensors, and optoelectronic measurement technology and system.

Jiabin Wang received his $\mathrm{BE}$ degree in chemical engineering and technology from Henan University of Urban Construction, Pingdingshan, China, in 2017. Currently, he is a postgraduate in optics engineering at the School of Physics, Dalian University of Technology. His current research interests include surface plasmon resonance sensors and biochemical sensing.

Zhenguo Jing received his $\mathrm{PhD}$ in optical engineering from the School of Physics, Dalian University of Technology, Dalian, China, in 2006. Currently, he is working in the School of Physics at Dalian University of Technology as an associated professor. His research 
interests are new photonic devices and Fabry-Perot interferometric optical fiber sensor and its applications.

Wei Peng received her doctoral degree in optical engineering from Dalian University of Technology, Dalian, China, in 1999. After spending four years in the Center for Photonics Technology within the
Department of Electrical Engineering at Virginia Polytechnic Institute and State University as a visiting scholar and postdoctor, she took a professor faculty position at Dalian University of Technology, China, in 2009. Currently, her research interests include fiber-optic sensors, surface plasmon resonance, and subwavelength optical design and fabrication. 